Original Article

\title{
Preliminary study of optimal measurement location on vibroarthrography for classification of patients with knee osteoarthritis
}

\author{
Susumu Ota, RPt, PhD ${ }^{1)^{*}}$, Akiko Ando, RPt, MA ${ }^{2)}$, Yusuke Tozawa, $\mathrm{MS}^{3)}$, \\ Takuya Nakamura, RPT ${ }^{2)}$, Shogo Okamoto, $\mathrm{PhD}^{4)}$, Takenobu SaKai, $\mathrm{PhD}^{5 \text {, }}$, \\ KAZUNORI HASE, $\mathrm{PhD}^{3)}$ \\ 1) Department of Rehabilitation and Care, Seijoh University: 2-172 Fukinodai, Tokai, Aichi 476-8588, \\ Japan \\ 2) Department of Physical Therapy, School of Health Sciences, Nagoya University, Japan \\ 3) Department of Mechanical Engineering, Graduate School of Science and Engineering, Tokyo \\ Metropolitan University, Japan \\ 4) Department of Mechanical Sciences and Engineering, Graduate School of Engineering, Nagoya \\ University, Japan \\ 5) Graduate School of Science and Engineering, Saitama University, Japan
}

\begin{abstract}
Purpose] The aims of the present study were to investigate the most suitable location for vibroarthrography measurements of the knee joint to distinguish a healthy knee from knee osteoarthritis using Wavelet transform analysis. [Subjects and Methods] Participants were 16 healthy females and 17 females with severe knee osteoarthritis. Vibroarthrography signals were measured on the medial and lateral epicondyles, mid-patella, and tibia using stethoscopes with a microphone while subjects stood up from a seated position. Frequency and knee flexion angles at the peak wavelet coefficient were obtained. [Results] Peak wavelet coefficients at the lateral condyle and tibia were significantly higher in patients with knee osteoarthritis than in the control group. Knee joint angles at the peak wavelet coefficient were smaller (more extension) in the osteoarthritis group compared to the control group. The area under the receiver operating characteristic curve on tibia assessment with the frequency and knee flexion angles was higher than at the other measurement locations (both area under the curve: 0.86 ). [Conclusion] The tibia is the most suitable location for classifying knee osteoarthritis based on vibroarthrography signals. Key words: Knee osteoarthritis, Vibroarthrography, Wavelet transform analysis
\end{abstract}

(This article was submitted Apr. 27, 2016, and was accepted Jul. 7, 2016)

\section{INTRODUCTION}

Knee osteoarthritis (OA) is a highly prevalent disease among the elderly and increases from middle age ${ }^{1,2)}$. Medial knee OA with varus deformity is 10 times more common than lateral knee $\mathrm{OA}^{3)}$, and females are more predisposed to it than males ${ }^{1,2)}$. The prevalence rate of knee OA among females increases from around the age of $40^{1)}$, and early diagnosis allows for treatment to be initiated even before symptoms present.

Vibroarthrography (VAG) is a noninvasive method of assessing cartilage degeneration or knee $\mathrm{OA}^{4-8)}$. A previous study ${ }^{8)}$ demonstrated that a high frequency area $(200-600 \mathrm{~Hz}$ ) obtained by fast Fourier transform (FFT) is a key feature of knee OA. Another study ${ }^{7}$ reported that a frequency of $50-150 \mathrm{~Hz}$ in patients with knee OA using FFT was higher than that in age-matched elderly. FFT does not take time information into account, and therefore FFT analysis cannot be applied to

*Corresponding author. Susumu Ota (E-mail: ota-s@seijoh-u.ac.jp)

(C2016 The Society of Physical Therapy Science. Published by IPEC Inc.

This is an open-access article distributed under the terms of the Creative Commons Attribution Non-Commercial No Derivatives (by-nc-nd) License $<$ http://creativecommons.org/licenses/by-nc-nd/4.0/>. 
investigate the relationship between knee joint motion and VAG signal frequency information. From this point of view, wavelet transform analysis is assumed to be a more suitable frequency analysis for VAG during tasks requiring knee joint motion because time is taken into account by this analytic method.

The accelerometer and microphone used to measure VAG signals from the knee joint were positioned around knee joint areas, such as the medial or lateral femoral condyles ${ }^{7,8)}$. The skin around the knee joint locations was expected to move during the tasks (gait, standing up, and walking up and down stairs). The most suitable location for the assessment of knee OA using VAG has not yet been reported. Only Shen et al. ${ }^{9)}$ reported positioning the accelerometer at the lateral and medial epicondyles, mid-patella, and tibia to investigate the origin of knee joint cartilage pathology using VAG. The optimal location for VAG measurements to classify normal knee and knee joint pathology, however, has not been evaluated.

The aims of the present study were to investigate the most suitable location for VAG measurements of the knee joint to distinguish a healthy knee from knee OA using Wavelet transform analysis. This is a preliminary study to classify knee OA. We conducted the study on extremely different populations, first in healthy young adults and then in patients with severe knee OA.

\section{SUBJECTS AND METHODS}

Participants were 16 healthy females (control group; age: $22 \pm 2$ years, height: $157 \pm 5 \mathrm{~cm}$, weight: $55 \pm 5 \mathrm{~kg}$ ) and 17 females with knee OA (OA group; age: $68 \pm 7$ years, height: $153 \pm 6 \mathrm{~cm}$, weight: $61 \pm 10 \mathrm{~kg}$ ). Subjects in the control group were recruited from a university, and exclusion criteria were as follows: history of previous lower extremity surgery, and previous injury that resulted in ligamentous laxity at the knee joint. The OA group was recruited from a local orthopedic clinic using a leaflet and poster on the bulletin board. Knee OA was diagnosed by an orthopedic surgeon according to the criteria of the American College of Rheumatology ${ }^{10)}$, and all patients with knee OA had medial knee OA (Kellgren-Lawrence classification; Grade III: 2, Grade IV: 15). All subjects were provided information regarding the nature of the study, and written informed consent was obtained, as required by the Research Ethics Committee of Seijoh University.

Subjects were seated in an armless chair $40 \mathrm{~cm}$ high and asked to stand up from the seated position taking $2 \mathrm{~s}$ to complete the movement. The standing movement was timed using a metronome. Measurements were performed on a randomly selected leg in the control group, and on the painful leg in the OA group. The standing-up task was performed three times, and the mean of each parameter (mentioned below) was used for the final analysis. The knee joint angle at three markers (femoral trochlear, lateral epicondyle, and lateral malleolus, Fig. 1) was measured using a two-dimensional (2D) camera system (Total Motion Coordinator Lite, Toso System Ltd., Japan) with a 60-Hz sample rate.

We used a custom-made VAG measurement system. General stethoscopes (Nursing scope double No.120, Kenzmedico Co., Ltd., Japan) were placed at the medial epicondyle, lateral epicondyle, patella, and tibia (Fig. 1) to obtain the VAG signals. One meter of rubber tube was connected to the head of the stethoscope and microphone (Size: $20 \mathrm{~mm} \times \mathrm{mm}$; flat area: 50 to $16 \mathrm{kHz}$, operational amplifier: OPA344 MicroAmplifier ${ }^{\mathrm{TM}}$ Series, Texas Instruments, Dallas, TX, USA). The VAG measurement system was confirmed to fall in a flat area from 100 to $2 \mathrm{kHz}$ using an electrodynamic shaker (Asahi Seisakusho, Ltd., Japan). The analogue VAG signals sampled at $50 \mathrm{kHz}$ were translated to digital data using an AD converter, and the signals were stored in a personal computer. The signals were then analyzed by MATLAB (R2013a, The MathWorks, Inc., Natick, MA, USA). A bandpass filter was used from 100 to $2 \mathrm{kHz}$, because muscle sound (mechanomyogram) interference is 0 to $100 \mathrm{~Hz}^{11)}$.

For analysis of the time-frequency properties of VAG, a wavelet transform analysis was carried out with MATLAB software. The mother wavelet used was "Morlet," and the analyzed frequency range was from 100 to $2 \mathrm{kHz}$. Contour maps of the wavelet-transformed data were obtained, and included time, frequency, and wavelet coefficient axes. In this study, two types of analysis were performed. One was a frequency analysis in which only frequency and wavelet coefficient values were used, and the other was a time-frequency analysis.

To perform the frequency analysis, the contour maps of the wavelet transform were projected onto the time axis. Then, 2D graphs were obtained to show the relationship between the frequency and wavelet coefficient. To quantify the VAG signal frequency properties, wavelet coefficients were normalized, and the number of subjects with normalized wavelet coefficients over 0.8 and frequency coefficients over 0.8 was compared. Frequencies were obtained with the analyzed 2D graphs.

A time-frequency analysis was used to investigate when and at what knee angle the VAG signals were emitted from knee joints, and the knee joint angle and frequency at the maximum wavelet coefficient were used to compare the OA and control groups.

The peak wavelet coefficient and knee angles at the peak coefficient were compared in four locations between groups using an unpaired t-test. Next, the area under the receiver operating characteristic (ROC) curve of the four assessment locations was obtained using the sensitivity and specificity of the peak frequency at the maximum wavelet coefficient and knee flexion angles, respectively. A p-value of less than 0.05 was considered statistically significant. 

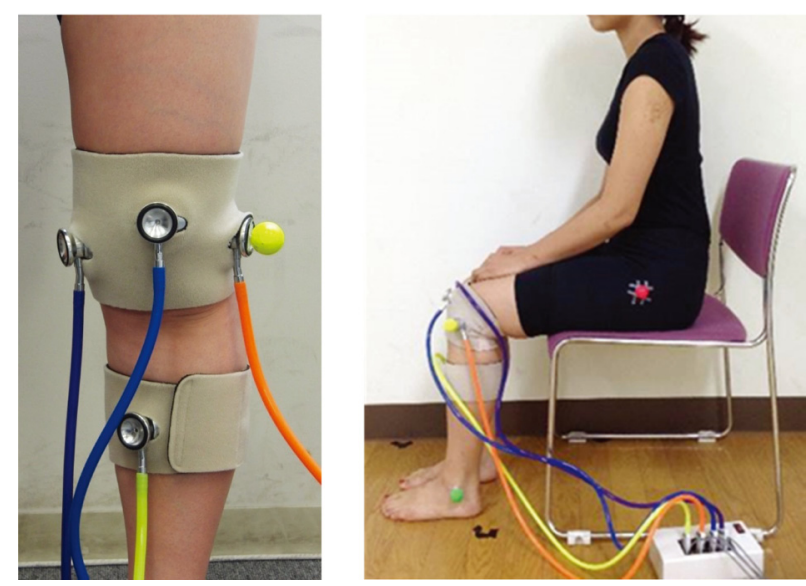

Fig. 1. Measurement setting for vibroarthrography of the knee joint

General stethoscopes were placed at the medial epicondyle, lateral epicondyle, patella, and tibia. The three color markers were set at femoral trochlear, lateral epicondyle, and lateral malleolus to assess the knee joint angle.

Table 1. Difference in vibroarthrography signal frequency at peak wavelet coefficient value between control and OA groups $(\mathrm{Hz})$

\begin{tabular}{lll}
\hline $\begin{array}{l}\text { Measurement } \\
\text { location }\end{array}$ & Control & \multicolumn{1}{c}{ OA } \\
\hline Medial & $151.8(64.0)$ & $218.4(138.2)$ \\
Lateral** $^{* *}$ & $164.6(82.4)$ & $250.6(86.3)$ \\
Patella & $155.6(43.1)$ & $180.5(77.1)$ \\
Tibia* & $122.2(53.6)$ & $176.9(91.2)$ \\
\hline
\end{tabular}

Medial: medial epicondyle; Lateral: lateral epicondyle. ${ }^{*} \mathrm{p}<0.05,{ }^{*} \mathrm{p}<0.01$. Values are shown as the mean \pm SD.

Table 2. Difference in knee joint angle at peak wavelet coefficient value between control and OA groups (degree)

\begin{tabular}{lcc}
\hline $\begin{array}{l}\text { Measurement } \\
\text { location }\end{array}$ & Control & OA \\
\hline Medial** & $50.8(15.4)$ & $34.6(17.0)$ \\
Lateral** & $59.8(17.4)$ & $39.4(19.0)$ \\
Patella & $55.9(23.6)$ & $41.6(16.4)$ \\
Tibia** & $62.3(17.9)$ & $37.5(15.1)$ \\
\hline
\end{tabular}

Medial: medial epicondyle; Lateral: lateral epicondyle.

$* * \mathrm{p}<0.01$. Values are shown as the mean \pm SD.
A. ROC curve of the frequency

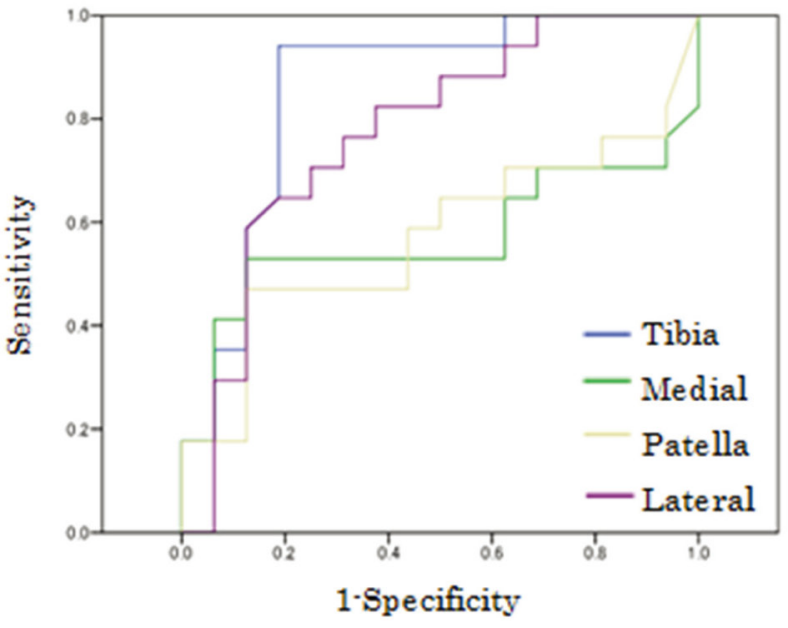

B. ROC curve of the angle

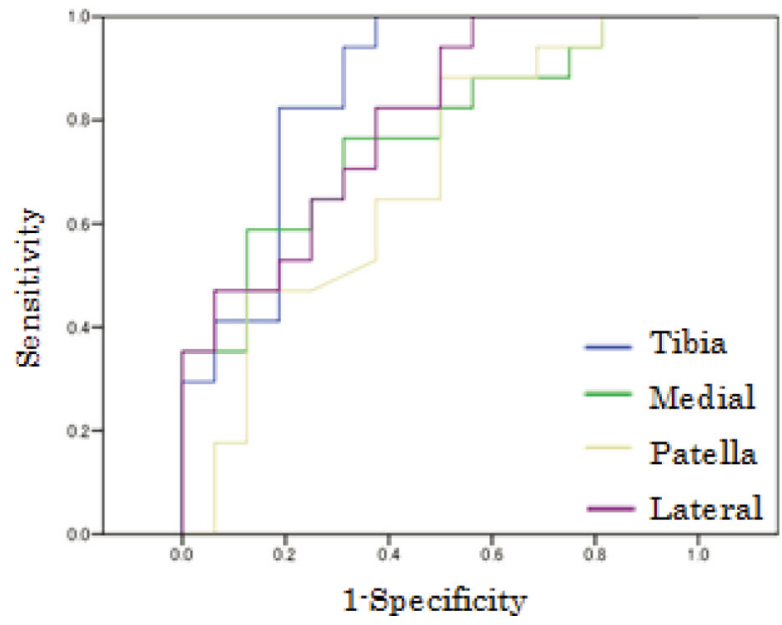

Fig. 2. ROC curve of frequency (A) and knee joint angle (B) at peak wavelet efficiency assessed at the four locations (medial, medial epicondyle; lateral, lateral epicondyle; patella; tibia)

\section{RESULTS}

Table 1 shows differences in the wavelet coefficient between groups. The peak wavelet coefficient at the lateral condyle and tibia in patients with knee OA was significantly higher than that in the control group. Table 2 shows knee joint angles at the peak wavelet coefficient between the control and OA groups. Angles in all locations of VAG measurement were significantly greater in the OA group than in the control group.

Figure 2 and Table 3 show the ROC curves and the values of the area under the curve (AUC) at the four assessment locations. The AUC values of the frequency and angle measurements were higher at the tibia than at the other locations. 
Table 3. Difference in AUC value between control and OA groups

\begin{tabular}{llllc}
\hline \multirow{2}{*}{$\begin{array}{l}\text { Measurement } \\
\text { location }\end{array}$} & \multicolumn{2}{c}{ Frequency } & \multicolumn{2}{c}{ Angle } \\
\cline { 2 - 5 } Medial & AUC & $95 \% \mathrm{Cl}$ & AUC & $95 \% \mathrm{Cl}$ \\
Lateral & 0.57 & $0.36-0.78$ & $0.77^{* *}$ & $0.60-0.93$ \\
Patella & $0.77^{* *}$ & $0.61-0.94$ & $0.80^{* *}$ & $0.65-0.95$ \\
Tibia & 0.57 & $0.37-0.78$ & 0.68 & $0.50-0.87$ \\
\hline
\end{tabular}

Medial: medial epicondyle; Lateral: lateral epicondyle; AUC: area under the curve; CI: confidence interval. $* \mathrm{p}<0.05, * * \mathrm{p}<0.01$

\section{DISCUSSION}

The peak VAG signal frequencies assessed at the lateral condyle and tibia in patients with OA were significantly higher than the those measured in the control group (Table 1), indicating that the signal frequency was higher in the OA group than in control. Extremely different joint conditions were compared between healthy young adults and patients with severe knee OA to investigate the optimal measurement location and angle of the knee joint, and to distinguish a healthy knee from a knee with OA. The knees of the patients in the OA group were considered to be in the final stage of OA (Kellgren-Lawrence classification; Grade III: 2, IV: 15), and we assumed that the VAG signal frequencies resulted from the impact between the femur and tibia when standing up. Angles correlating with the peak VAG signal intensity at all measurement locations were significantly smaller in the OA group (less knee joint extension) than in the control group. We considered that the VAG signals related to joint friction were associated with the location of the knee joint cartilage degeneration and knee joint kinematics with the combination of sliding and rolling. Because there were no such data in the present study, however, we cannot discuss the relationship between VAG signals and knee kinematics.

The VAG results on the lateral epicondyles were assumed to have resulted from friction between the lateral epicondyle and iliotibial band, with running being the cause of iliotibial band friction syndrome ${ }^{12}$ ). McCoy et al. indicated that VAG signals due to friction with the iliotibial band was observed at knee flexion angles between $19^{\circ}$ and $55^{\circ}$. The angle at the peak wavelet coefficient value averaged 39.4 \pm 19.0 degrees (Table 1), and both ranges were similar. Therefore, we considered that VAG signals measured at the lateral epicondyle might include signals representing friction between the lateral epicondyle and iliotibial band.

Two previous studies ${ }^{8,9)}$ investigated three or four assessment locations to obtain the origin of knee joint VAG signals. No studies to date have compared knee-joint location for VAG assessment during a weight-bearing task to investigate VAG features of knee OA. Based on the AUC analysis (Table 3), the tibia is the most suitable location to distinguish normal knee joints from knee joints with OA through assessing the location of VAG. Two anatomic reasons were assumed to underlie this finding: the tibia attached to the accelerometer is the flattest bone and the tissue is thinner compared to that on the medial epicondyle, lateral epicondyle, and patella. Therefore, the noise on the tibia might be lower than that at the other locations.

Previous studies described a difference in the frequency based on $\mathrm{FFT}^{7,8)}$ or signal variations (jitter and shimmer analysis) ${ }^{13)}$ between normal knee and knees with OA. So far, however, there are no true VAG data between the femur and tibia, because it is impossible to measure areas just next to impact in vivo. Therefore, one should first investigate the conditions for obtaining significant differences between healthy knees and knees with OA. Based on our findings, the tibia is the most suitable location for distinguishing VAG signals of a healthy knee joint from those of knees joints with OA.

The present study has several limitations. First, the origin of the VAG, such as the femoral tibia joint including the meniscus or the patellar femoral joint, could not be distinguished in the present study because the VAG included the whole vibration around the knee joint during the standing task. We did not compare validity data as in some previous VAG studies. In the future, a basic animal VAG study using an OA model should be performed as a validity study. Second, the subjects were young healthy adults and patients at the final stage of knee OA. Therefore, we cannot discuss the progression of cartilage degeneration. Alternatively, a quantitative cartilage assessment like T2 mapping on magnetic resonance images could be conducted to validate VAG in knee OA.

In conclusion, VAG signals were assessed at four locations (lateral and medial epicondyles, mid-patella, and tibia) to investigate the optimal place to assess healthy and osteoarthritic knees. The tibia was the most suitable location to distinguish the two conditions, and the frequency and knee flexion angle at the peak wavelet efficiency assessed on the tibia in patients with knee OA were higher and lower, respectively, than the values in the healthy population. 


\section{ACKNOWLEDGEMENTS}

The present study was partially supported by Suzuken Memorial Foundation (12-0006) and a Grant-in-Aid for Scientific Research (B) (15H03047).

\section{REFERENCES}

1) Yoshimura N, Muraki S, Oka H, et al.: Prevalence of knee osteoarthritis, lumbar spondylosis, and osteoporosis in Japanese men and women: the research on osteoarthritis/osteoporosis against disability study. J Bone Miner Metab, 2009, 27: 620-628. [Medline] [CrossRef]

2) Lawrence RC, Felson DT, Helmick CG, et al. National Arthritis Data Workgroup: Estimates of the prevalence of arthritis and other rheumatic conditions in the United States. Part II. Arthritis Rheum, 2008, 58: 26-35. [Medline] [CrossRef]

3) Ahlbäck S: Osteoarthrosis of the knee. A radiographic investigation. Acta Radiol Diagn (Stockh), 1968, (Suppl 277): 277, 7-72. [Medline]

4) Chu M, Gradisar I, Mostardi R: A noninvasive electroacoustical evaluation technique of cartilage damage in pathological knee joint. Med Biol Eng Comput, 1978, 16: 437-442. [Medline] [CrossRef]

5) Frank CB, Rangayyan RM, Bell GD: Analysis of knee joint sound signals for non-invasive diagnosis of cartilage pathology. IEEE Eng Med Biol Mag, 1990, 9: 65-68. [Medline] [CrossRef]

6) Kernohan WG, Barr DA, McCoy GF, et al.: Vibration arthrometry in assessment of knee disorders: the problem of angular velocity. J Biomed Eng, 1991, 13: 35-38. [Medline] [CrossRef]

7) Tanaka N, Hoshiyama M: Articular sound and clinical stages in knee arthropathy. J Musculoskelet Res, 2011, 14: 11500061-11500069. [CrossRef]

8) McCoy GF, McCrea JD, Beverland DE, et al.: Vibration arthrography as a diagnostic aid in diseases of the knee. A preliminary report. J Bone Joint Surg Br, 1987, 69: 288-293. [Medline]

9) Shen Y, Rangayyan RM, Bell GD, et al.: Localization of knee joint cartilage pathology by multichannel vibroarthrography. Med Eng Phys, 1995, 17: 583-594. [Medline] [CrossRef]

10) Altman R, Asch E, Bloch D, et al. Diagnostic and Therapeutic Criteria Committee of the American Rheumatism Association: Development of criteria for the classification and reporting of osteoarthritis. Classification of osteoarthritis of the knee. Arthritis Rheum, 1986, 29: 1039-1049. [Medline] [CrossRef]

11) Zhang YT, Rangayyan RM, Frank CB, et al.: Adaptive cancellation of muscle contraction interference in vibroarthrographic signals. IEEE Trans Biomed Eng, 1994, 41: 181-191. [Medline] [CrossRef]

12) Orchard JW, Fricker PA, Abud AT, et al.: Biomechanics of iliotibial band friction syndrome in runners. Am J Sports Med, 1996, 24: 375-379. [Medline] [CrossRef]

13) Kim KS, Seo JH, Song CG: An acoustical evaluation of knee sound for non-invasive screening and early detection of articular pathology. J Med Syst, 2012, 36: 715-722. [Medline] [CrossRef] 